\title{
Construction and performance of a drop cell for the nephelometric determination of sulfur dioxide
}

\author{
Márcio R. Milani*, Arnaldo A. Cardoso \\ Instituto de Química UNESP, Departamento Química Analítica, C.P. 355 CEP 14800-900, Araraquara, SP, Brazil
}

Received 8 May 2002; received in revised form 13 September 2002; accepted 18 September 2002

\begin{abstract}
A nephelometric technique based on a liquid drop is described for the measurement of atmospheric sulfur dioxide. A 40- $\mu 1$ drop of barium chloride and hydrogen peroxide solution is suspended in a flowing-air sampling stream. The sulfur (IV) collected is oxidized to sulfur (VI) and finally precipitated as barium sulfate. Nephelometric detection of drop is achieved by an appropriate arrangement consisting of an optical fiber contacting the drop and a photodiode placed at $90^{\circ}$ relative to the fiber. The design and characteristics of this drop-based gas sensor system are described. The analytical response, as photocurrent, is proportional to the product of the sampling period and the sulfur dioxide concentration. The detection limit is ca. $1.1 \mathrm{mg} \mathrm{m}^{-3}$ for a 10 -min sampling time. The present technique is fairly rapid and simple, uses a small amount of reagent and is set up with low-cost equipment, making this system economically viable.
\end{abstract}

(c) 2002 Elsevier Science B.V. All rights reserved.

Keywords: Nephelometry; Sulfur dioxide determination; Drop analysis

\section{Introduction}

Sulfur dioxide $\left(\mathrm{SO}_{2}\right)$ is one of the most important atmospheric pollutants and its emission is directly related to combustion processes, such as the consumption of fossil fuels and burning of biomass. Sulfur dioxide can also be formed in the atmosphere by the oxidation of reduced sulfur compounds. Sulfur gases are important in atmospheric chemistry due to their role in the genesis of acid precipitation and degradation of atmos-

\footnotetext{
*Corresponding author.

E-mail addresses: marcmila@vetorialnet.com.br (M.R. Milani), acardoso@iq.unesp.br (A.A. Cardoso).
}

pheric visibility. The measurement of atmospheric $\mathrm{SO}_{2}$ has received considerable attention and a number of analytical methods have been developed [1-4]. The preference is for simple, field-deployable and affordable methods. Most such methods for the estimation of levels of $\mathrm{SO}_{2}$ in gaseous samples comprise of two steps, collection and storage of $\mathrm{SO}_{2}$ from air in a medium and its subsequent determination by a convenient analytical technique. Sulfur dioxide can be absorbed in tetrachloromercurate [5], formaldehyde [6] or hydrogen peroxide [7] solutions. In the first two cases, $\mathrm{SO}_{2}$ is preserved as $\mathrm{S}(\mathrm{IV})$, while with $\mathrm{H}_{2} \mathrm{O}_{2}$ it is oxidized to $\mathrm{S}(\mathrm{VI})$. The determination can be made by using ion chromatography [8] or 


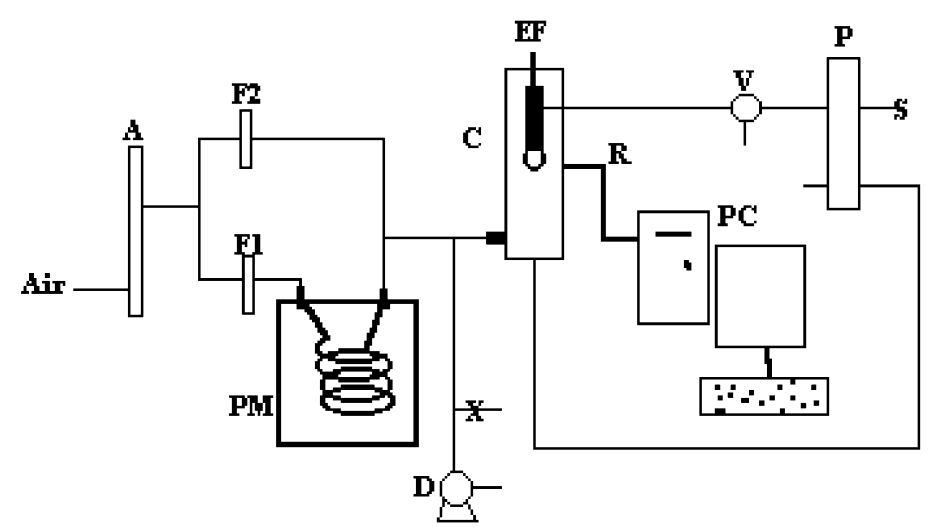

Fig. 1. Schematic diagram of the system to generate sulfur dioxide standard samples and to acquire the signal. (A) columns for air purification; $\left(\mathrm{F}_{1,2}\right)$ flowmeters; $(\mathrm{PM})$ gas generator; $(\mathrm{C})$ sampling chamber; $(\mathrm{R})$ optical fiber; (EF) optical fiber that conducts the light beam from the spectrophotometer to drop head; (D) vacuum pump; (P) peristaltic pump; (S) sampling solution; (V) threeway valve; and (PC) computer to record data.

an optical technique, such as para-rosaniline [5,6], barium sulfate turbidimetry [9], or the methylthymol blue colorimetric method [10]. The analytical process of fixation, storage and determination of $\mathrm{SO}_{2}$ requires laborious procedures, which are timeconsuming and can also be a source of additional errors.

One alternative to traditional gas analysis was proposed in the mid-1990s. A droplet of solution coupled with an optical fiber can be used to collect a gaseous analyte while the droplet is simultaneously optically excited to determine the analyte concentration [11-15]. The liquid drop is reproducible, renewable and has a defined volume without containment walls. These features make such a system suitable for determining gaseous analytes with the added advantages of simplicity and measurement near real time.

Barium sulfate precipitation is the classic way to analyze sulfate, using either turbidimetry or nephelometry. Once $\mathrm{SO}_{2}$ can be oxidized to sulfate, then it can be applied for the measurement of $\mathrm{SO}_{2}$. Although the method exhibits relatively poor precision and accuracy at low levels of $\mathrm{SO}_{2}$, it has been widely used because only simple chemistry and equipment normally present in any chemistry laboratory are required. Turbidimetry is based on the measurement of the light intensity absorbed by small particles in suspension, similar to absorb- ance, while in nephelometry the intensity of the light scattered by the particles is measured and can be related to the solid concentration. Nephelometry is used for the measurement of the concentration of very dilute suspensions, while for more concentrated suspensions turbidimetry should be employed [16]. Liu and Dasgupta [17] have shown the potential of the liquid drop as an optical cell for the determination of sulfate via the precipitation reaction between barium and sulfate ions in a flow injection analysis (FIA) system. This is a windowless cell system, and consequently the error caused by deposition of $\mathrm{BaSO}_{4}$ does not occur. Deposition in ducts and on the walls of the cuvet is said to be one of main causes of errors in sulfate analysis by FIA [18]. In the present work, to extend the application of the photometric drop cell for the determination of sulfur dioxide in air, a simple instrument to measure the sulfur dioxide absorbed by a drop of hydrogen peroxide and barium chloride solution was developed. The barium sulfate precipitated was determined using a drop as a nephelometric cell.

\section{Experimental}

\subsection{Standard gas generation and test arrangement}

The standard gas samples were generated according to Dasgupta and Dong [19]. The sam- 
pling system and the test gas-generation device used are represented in Fig. 1. Compressed house air is purified by passage through a column (A), containing sequential beds of silica gel and activated carbon. The pure air is then divided into two streams. One portion of air, with flow controlled by a flowmeter $\left(\mathrm{F}_{1}\right)$ with maximum capacity of $250 \mathrm{ml} \mathrm{min}{ }^{-1}$ (Gilmont Instruments, Racine, WI), is directed through the gas generator based on a hydrophobic microporous membrane tube (PM) immersed in $10 \mathrm{mmol}^{-1} \mathrm{NaHSO}_{3}$ solution, buffered with $75 \mathrm{mmol}^{-1}$ potassium acid phthalate. A stable concentration of $\mathrm{SO}_{2}$ is generated by equilibration of the air with aqueous hydrogen sulfite solution through the microporous membrane tube. All measurements were made after allowing the system to equilibrate for several hours. A second pure air stream, controlled by a flowmeter $\left(\mathrm{F}_{2}\right)$ with maximum capacity of $250 \mathrm{ml} \mathrm{min}{ }^{-1}$, is used for dilution of the exit gas from the generator. This flow is used to feed the sampling chamber (C), and the non-sampled fraction of the standard gas is discarded with the aid of a vacuum pump (D). The actual $\mathrm{SO}_{2}$ concentration is determined just prior to experiments. The exit air stream is passed through a fritted midget bubbler containing a solution of dilute hydrogen peroxide. The $\mathrm{SO}_{2}$ is oxidized to sulfuric acid. The acid is then titrated by $\mathrm{NaOH}$ standard solution following a procedure described elsewhere [20]. A second identical bubbler in series checks the quantitative collection of $\mathrm{SO}_{2}$.

An analog variable-speed peristaltic pump (P) (Ismatec, model 7335-50) transports the solution (S) from a beaker to the drop head inside the sampling chamber. The volume of the drop is defined when the flow of solution to the sampling chamber is stopped by a three-way valve (V).

\subsection{Sampling chamber and sensor arrangement}

The sampling chamber and sensor are shown in Fig. 2. The silica optical fiber (EF) $(1000-\mu \mathrm{m}$ core, fused silica; Ensign-Bickford Optics, Avon, CT) conducts the light beam from the exit slit of a spectrophotometer (Procyon SC90) to the drop. An ordinary tungsten filament lamp is used as a source of radiation. The light scattered by the

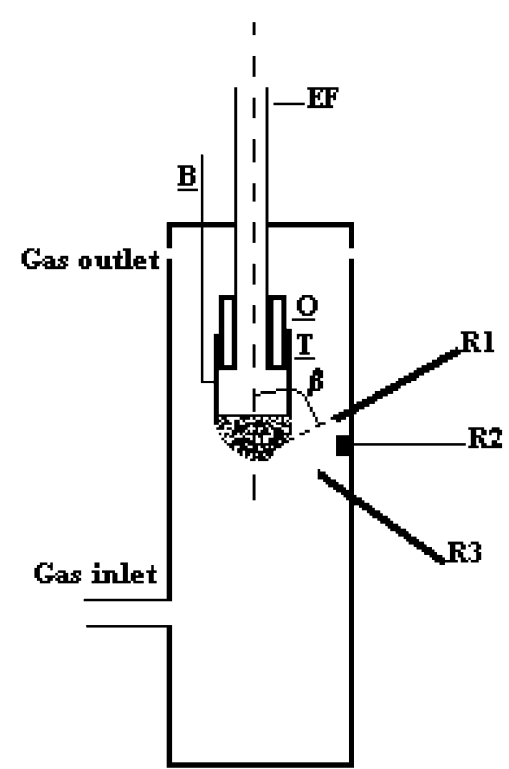

Fig. 2. Details of the sampling chamber and drop head. The angles $(\beta)$ tested were $30^{\circ}, 90^{\circ}$ and $150^{\circ}$, being formed by the crossing of the axis of the drop head and the optical fiber (R1, R2 and R3, respectively).

particles in suspension is collected by an optical fiber (R) and transported to the photodiode. The photodiode output is connected to an electronic current detector [21], which is interfaced to a personal computer (PC) with a MQI-96A board (Microquimica Ltda, Brazil). The scattered light is measured and recorded as electrical current. The sampling chamber consists of a black PVC tube (12-mm i.d. and 70-mm length). The optical fiber (EF) is positioned inside a Teflon tube (T) (4.48$\mathrm{mm}$ i.d. and $8-\mathrm{mm}$ length). The end of the optical fiber is $2 \mathrm{~mm}$ above the tip of tube (T). A PTFE capillary (B) (1.48-mm i.d. and 3-mm o.d.) is inserted into the tube $(\mathrm{T})$ to deliver the solution and to form the drop. The escape of solution from the top, due to the capillary effect, is prevented using O-rings (O). Since light is scattered in all directions and the incident light travels straight across the drop, the optical fiber (R) should be positioned so as to minimize the generation of stray light. The optical fiber ( $R$ ) was positioned at different angles $(\beta)$ with the tube $(T)$, until optimal conditions were achieved. 


\subsection{Chemicals}

All the chemicals were reagent grade quality. Deionized water was used throughout. The barium sulfate suspensions were prepared with different aliquots of $2.1 \times 10^{-3} \mathrm{~mol} \mathrm{l}^{-1} \mathrm{H}_{2} \mathrm{SO}_{4}$ added to $0.5 \mathrm{ml}$ of $25 \%(\mathrm{w} / \mathrm{v}) \mathrm{BaCl}_{2}$, followed by dilution to $100 \mathrm{ml}$. The suspension was labeled with the final sulfate concentration. Each suspension was stirred for $5 \mathrm{~min}$ at room temperature. The gas generation solution was prepared with $1.26 \mathrm{~g}$ of sodium sulfite and $15.32 \mathrm{~g}$ of potassium acid phthalate and made up to $1000 \mathrm{ml}$ with deionized water. The concentration of the solution was 10 mmol $1^{-1}$ sodium sulfite at $\mathrm{pH} 4.3$. The absorbing reagent was freshly prepared with $0.3 \%(\mathrm{v} / \mathrm{v})$ hydrogen peroxide and $5 \%(\mathrm{w} / \mathrm{v})$ barium chloride.

\section{Results and discussion}

\subsection{Sensor parameters}

\subsubsection{Signal dependence on drop volume and wavelength of source light}

As shown in previous work [17], the scattered light depends on the drop volume. During a cycle in which a drop grows and falls, the photocurrent registered shows a maximum and minimum. It occurs because the drop acts like a lens that continuously changes its focus. A first experiment was carried out with a $\mathrm{BaSO}_{4}$ suspension drop growing and falling while the signal was monitored. Monochromatic light at $525 \mathrm{~nm}$ was used for excitation and the photodiode was placed at $90^{\circ}$ to the emitting fiber (position R2 in Fig. 2). Since the peristaltic pump keeps the liquid flow rate constant, the drop volume is related to the flow time. The solution flow was kept constant at $13.3 \mu \mathrm{l} \mathrm{min}{ }^{-1}$ and the signal registered could be related to a known drop volume. As described in previous work $[14,15]$, the optimum volume is the one that gives the best analytical signal and minimum standard deviation. Under the conditions described here, a drop volume of $40 \mu \mathrm{l}$ was chosen as optimum for further work, since this gave a good analytical signal and drop stability.

The theory of the scattering of light by very small particles was formulated by Lord Rayleigh
[22] approximately 130 years ago. The intensity of light scattered from particles increases rapidly as the wavelength $(\lambda)$ decreases by a factor $\lambda^{-4}$. However, the photodiode used in this work is less sensitive to low wavelengths, and hence an optimum wavelength should exist for detection. The energy of the source lamp also decreases at low wavelengths, and thus various wavelengths must be tested. The experiments were performed using $100 \mathrm{mg}^{-1}$ sulfate suspension to form a drop of $40 \mu \mathrm{l}$ and the photodiode was placed at $90^{\circ}$ to the emitting fiber. The wavelength of the radiant energy was increased in 25-nm steps from 400 to 700 $\mathrm{nm}$, while the photocurrent was recorded. This experiment showed that $450 \mathrm{~nm}$ is suitable for analytical purposes; it gives one of the highest photocurrent values in the wavelength range evaluated, as observed in Fig. 3. However, this experiment was limited by the light source, and the use of another light source could extend the range of measurement well into the ultraviolet region. As can be observed in Fig. 3, the intensity of light scattering increases in the direction of lower wavelength.

\subsubsection{Angular distribution of scattered light}

The angular distribution of scattered light is not uniform [23] and depends on specific properties of the suspension studied. To reach the best optical fiber position (R, Fig. 2), drops of suspensions containing six different sulfate concentrations $(0-$ $10 \mathrm{mg}^{-1}$ ) were tested, recording the photocurrent generated by a 450 -nm light beam. In the experiments, the optical fiber $(\mathrm{F})$ was positioned at the following angles: $30^{\circ}, 90^{\circ}$ and $150^{\circ}$. The angle is defined by the crossing of the axis of the optical fiber (EF) with the optical fiber (R) axis. Table 1 shows the parameters of the lines that best fit sulfate concentration vs. photocurrent, for different angles experienced. Considering the slope of the adjusted line as representative of the system sensitivity, and the correlation index $\left(R^{2}\right)$ as representative of the global quality of the results, the assembly using $150^{\circ}$ is more attractive than $90^{\circ}$. However, the $90^{\circ}$ angle permits a new design of sampling chamber, without the optical fiber (R) and with the photodiode positioned directly on the wall of the sampling chamber, enabling optimum 


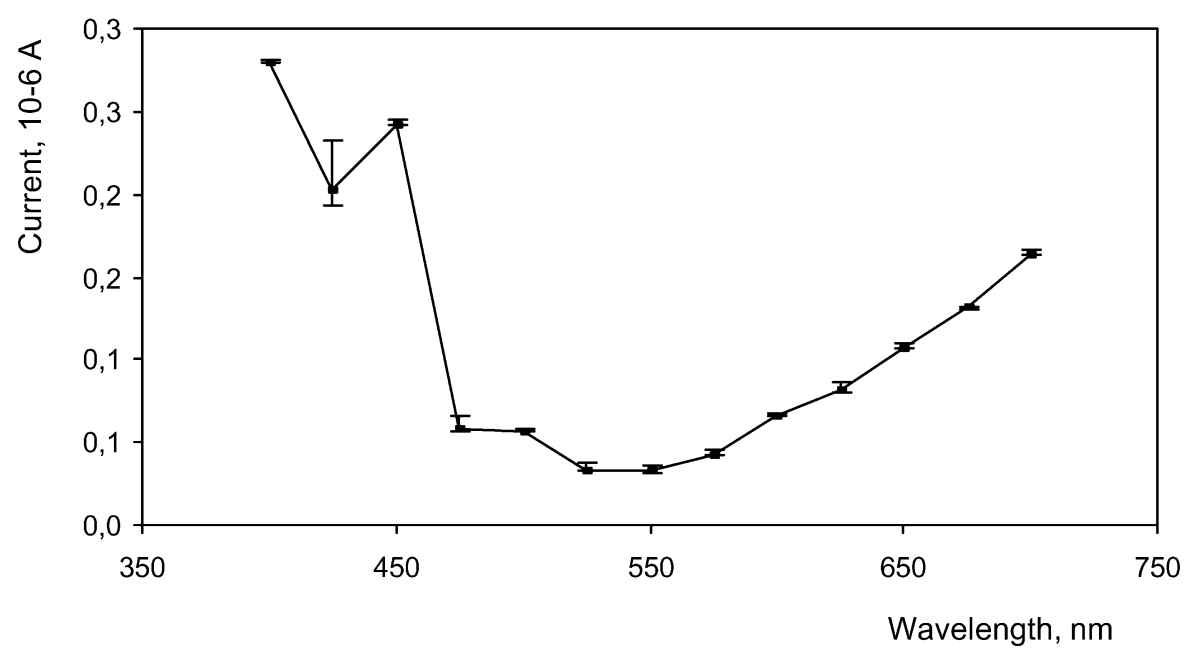

Fig. 3. Absorption spectrum of barium sulfate suspension. Photocurrent measured from light scattered by a $40-\mu 1 \mathrm{drop}$ of $100 \mathrm{mg}$ $1^{-1}$ sulfate suspension. Errors bars at $3 \times$ S.D.

generation of the scattered light. For this reason, an angle of $90^{\circ}$ was used in subsequent experiments.

\subsubsection{Variation of current intensity according to sulfate concentration}

$\mathrm{Six} \mathrm{BaSO}_{4}$ suspensions $\left(0-80 \mathrm{mg} \mathrm{1^{-1 }}\right)$ were prepared and used to form a drop of $40 \mu \mathrm{l}$, and a light beam of $450 \mathrm{~nm}$ was used as radiant energy for the nephelometric measurements. The analytical signal is linear with the concentration of $\mathrm{BaSO}_{4}$. For these conditions, the analytical curve obtained is:

$$
\begin{aligned}
I(\mu A)= & (1.24+0.06) \times 10^{-4}\left[\mathrm{SO}_{4}^{2-}\right]\left(\mathrm{mg} \mathrm{l}^{-1}\right) \\
& +(2.85+0.03) \times 10^{-2}\left(R^{2}=0.989\right)
\end{aligned}
$$

The results show that this detection system with a drop of $40 \mu \mathrm{l}$ can be effectively used to determine barium sulfate in suspension nephelometrically at $\lambda=450 \mathrm{~nm}$.

\subsection{Experiments using gaseous samples}

The analysis protocol in these experiments consisted of the following steps:

a. Formation of a droplet $(40 \mu \mathrm{l})$ with absorbing reagent;

b. Recording the photocurrent;

c. Sampling of $\mathrm{SO}_{2}$ for a fixed time and flow rate;

d. Flushing the sampling chamber with pure air for $10 \mathrm{~s}$;

e. Waiting 3 min until the formation of $\mathrm{BaSO}_{4}$ is complete and reading the photocurrent; and

f. Reestablishing liquid flow to form a new drop and restart the process.

\subsubsection{Effect of sampling time}

In air analyses, sampling time is an important parameter, since the detection limit and collection

Table 1

Equations for analytical curves

\begin{tabular}{rlr}
\hline Angle, $\beta\left(^{\circ}\right)$ & Equation & $R^{2}$ \\
\hline 30 & $I=\left(0.011+3.4 \times 10^{-5}\right)+(6.2+0.6) \times 10^{-5} C$ & 0.965 \\
90 & $I=\left(0.013+9.2 \times 10^{-5}\right)+(8.8+1.5) \times 10^{-5} C$ & 0.924 \\
150 & $I=\left(0.023+3.0 \times 10^{-4}\right)+(40.1+3.6) \times 10^{-5} C$ & 0.969
\end{tabular}

Sulfate concentration $\left(C, \mathrm{mg}^{-1}\right)$ vs. photocurrent $(I, \mu \mathrm{A})$ for different angles $\left(\beta,^{\circ}\right)$. 


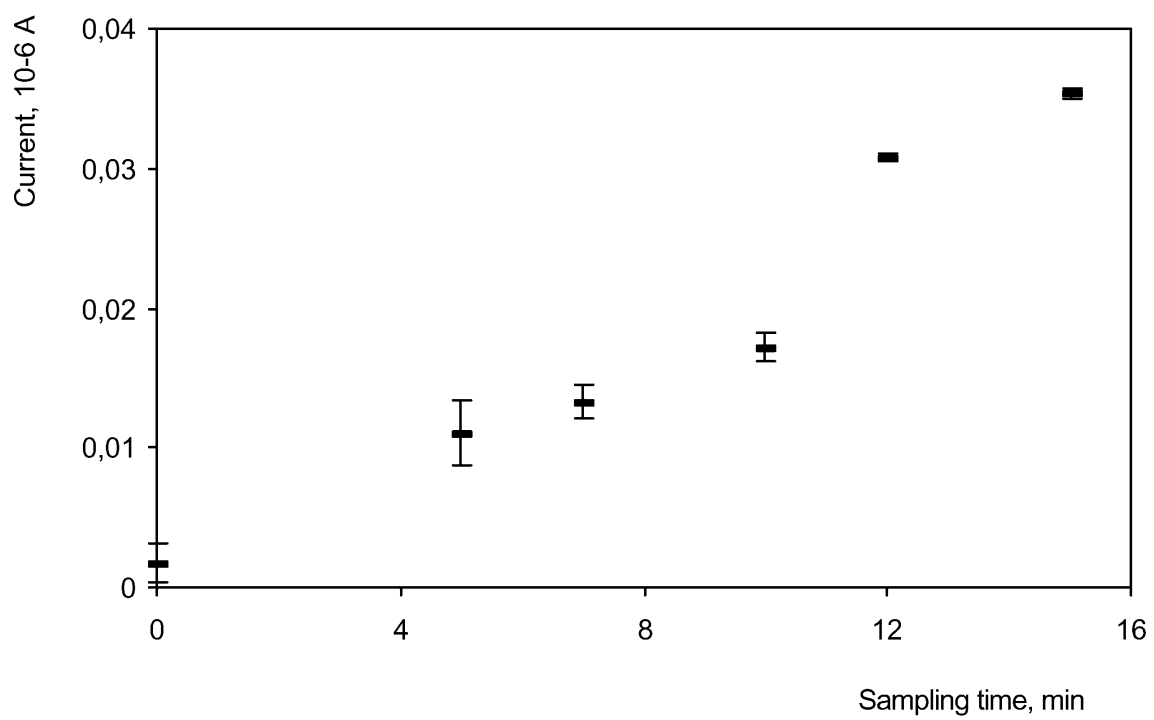

Fig. 4. Effect of sampling time. Conditions: $\lambda$, $450 \mathrm{~nm}$; drop volume, $40 \mu \mathrm{l}$; absorbing solution, $0.3 \%(\mathrm{v} / \mathrm{v}) \mathrm{H}_{2} \mathrm{O}_{2}$ and $5 \%(\mathrm{w} / \mathrm{v}$ ) $\mathrm{BaCl}_{2}$; gaseous sampling concentration, $8.3 \mathrm{mg} \mathrm{SO}_{2} \mathrm{~m}^{-3}$; sampling flow rate, $100 \mathrm{ml} \mathrm{min}{ }^{-1}$. Errors bars at $3 \times \mathrm{S} . \mathrm{D}$.

capacity, for example, are dependent on sampling time. In this experiment, a drop of $40 \mu \mathrm{l}$ of absorbing reagent is formed and kept stationary. A gaseous sample containing $8.3 \mathrm{mg} \mathrm{SO}_{2} \mathrm{~m}^{-3}$ is

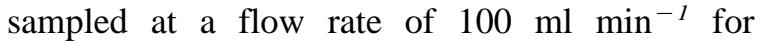
different time intervals. The photocurrent stabilizes 2 min after the end of the sampling. The analytical signal is the difference between the photocurrent recorded $3 \mathrm{~min}$ after the end of sampling and that at the beginning of sampling. Since the flow through the porous tube and the permeation rate are kept constant, a longer sampling time results in a greater amount of $\mathrm{SO}_{2}$ sampled. Considering that the analytical signal is related to the concentration of barium sulfate formed (i.e. the amount of $\mathrm{SO}_{2}$ sampled), a linear relationship between signal and sampling time is expected. The relationship between sampling time and photocurrent obtained in the experiments can be observed in Fig. 4. A sampling time longer than 10 min makes the signal increase rapidly. This behavior probably results for two reasons:

a. Evaporation of part of the solution would increase the sulfate concentration in the solution. Cardoso and Dasgupta [12] have showed experimentally the influence of evaporation over the analytical signal; the effect of sample relative humidity was also previously described [24].

b. Sulfate precipitation in a windowless cell is possibly influenced by the amount of pre-existing solids. The mechanism and kinetics of the formation of $\mathrm{BaSO}_{4}$ indicate that the first stage of precipitation involves nucleation and growth, whereas the second stage involves only growth. During the growth period, changes may occur as flocculation or agglomeration, resulting in modification of scattering properties. An enormous variation in the angular distribution of the scattered light has been observed between small, medium and large particles [25]. Unfortunately, the particle size in this experiment cannot be completely controlled, and for this reason measurement should be made either during the first stage for low concentrations of $\mathrm{SO}_{2}$, or the second stage for high concentrations.

Measurement close to the change point of the process has to be avoided, and for these experiments using gaseous samples it is advisable to avoid sampling times greater than $8 \mathrm{~min}$. Alcohol or other reagents may be added in an effort to minimize the solubility of the particles, stabilize the particle size and prevent particle agglomera- 
tion. The use of the conditioning agents ethanol and glycerol were examined, with the conditioning agent added to the reagent used for formation of the droplet. The results are worse than when the conditioning agent is absent. This can be explained because the process depends upon the oxidation of $\mathrm{S}(\mathrm{IV})$ to S(VI), and most of the reaction mechanisms published suggest oxidation involving radicals [26]. The radical scavengers ethanol and glycerol probably inhibit the overall S(IV) oxidation process. The fact that glycerol and ethanol increase the viscosity and prevent surface layer remixing may also be taken into account as being responsible for this behavior.

\subsubsection{Response characteristics}

Experiments were conducted under the analytical conditions previously determined to be the best for $\mathrm{SO}_{2}$. A drop of $40 \mu \mathrm{l}$ of absorbing reagent was formed and kept stationary and a 450-nm light beam was used as the excitation source. A sampling time of $3 \mathrm{~min}$ at a flow rate of $130 \mathrm{ml}$ $\min ^{-1}$ was used. The analytical signal is taken as the difference between the initial photocurrent at the beginning of the experiment and 3 min after the cessation of sampling. Standard gas mixtures were obtained by diluting the stream containing $\mathrm{SO}_{2}$ with an appropriate flow rate of clean air. The results show a linear relationship within a $\mathrm{SO}_{2}$ concentration range of $0-15 \mathrm{mg} \mathrm{m}^{-3}$ :

$$
\begin{gathered}
I(\mu A)=(1.35+0.05) \times 10^{-3}\left[\mathrm{SO}_{2}\right]\left(\mathrm{mg} \mathrm{m}^{-3}\right) \\
+(1.79+0.43) \times 10^{-3}, R^{2}=0.997
\end{gathered}
$$

The lowest concentration tested was $2 \mathrm{mg} \mathrm{SO}$ $\mathrm{m}^{-3}$ and the detection limit, based on the criterion of three times the standard deviation of the blank, is better than $1.1 \mathrm{mg} \mathrm{SO}_{2} \mathrm{~m}^{-3}$. However, previous experiment suggests that the detection limit can be readily improved by using a sampling time longer than $3 \mathrm{~min}$ and shorter than $8 \mathrm{~min}$.

\section{Conclusions}

The potential of an in situ droplet-based $\mathrm{SO}_{2}$ sensor was demonstrated here. The $\mathrm{SO}_{2}$ is collected by a drop of solution containing $\mathrm{H}_{2} \mathrm{O}_{2}$ and $\mathrm{BaCl}_{2}$. The sulfur dioxide is oxidized by hydrogen peroxide to sulfuric acid and the sulfate is precipitated as barium sulfate. Nephelometric detection of the drop is achieved with an appropriate arrangement of a light source and photodiode around the drop. While the present arrangement has reasonable sensitivity, and is thus a convenient technique for the determination of $\mathrm{SO}_{2}$, further improvements could lead to detection systems with greater capability. The use of a UV or blue LED as a source could work as well as or better than the light beam from the spectrophotometer used in this work, also simplifying the equipment.

The analytical method proposed is rapid and simple, uses a small amount of reagent and requires low-cost equipment, making the system economically viable for a greater range of potential users.

\section{Acknowledgments}

This project was supported by CNPq (National Science Foundation of Brazil) and FAPESP (Science Foundation of São Paulo State). MRM acknowledges financial support from CAPES (National Professor Improvement Foundation of Brazil).

\section{References}

[1] S. Bruckenstein, K.A. Tucker, P.R. Gifford, Anal. Chem. 52 (1980) 2396.

[2] F. Bruner, A. Liberti, M. Possanzini, I. Allegrini, Anal. Chem. 44 (1972) 2070.

[3] D.W.T. Griffith, G.J. Schuster, Atmos. Chem. 5 (1987) 59.

[4] R.J. Ferec, D.A.J. Hegg, Geophys. Res. 98 (1993) 23435.

[5] P.W. West, G.C. Gaeke, Anal. Chem. 28 (1956) 1816.

[6] P.K. Dasgupta, K. DeCesare, J.C. Ullrey, Anal. Chem. 52 (1980) 1912.

[7] M.D. Thomas, J.O. Ivie, T.C. Fitt, Ind. Eng. Chem. Anal. Ed. 18 (1946) 383.

[8] E. Sawicki, J.D. Mulik, E. Wittgenstein (Ed.), Ion Chromatography of Environmental Pollutants, vol. 1, Ann Arbor Science Publishers, Ann Arbor, MI, 1978.

[9] Standard Test for Sulfate Ion in Water, D516-90, American Society for Testing and Material, West Conshohocken, PA, 1997.

[10] G. Colovos, M.R. Panesar, E.P. Parry, Anal. Chem. 48 (1976) 1693. 
[11] A.A. Cardoso, P.K. Dasgupta, Anal. Chem. 67 (1995) 2562.

[12] A.A. Cardoso, H. Liu, P.K. Dasgupta, Talanta 44 (1997) 1099.

[13] E.A. Pereira, P.K. Dasgupta, Int. J. Environ. Anal. Chem. 66 (1997) 201.

[14] M.R. Milani, J.A. Gomes Neto, A.A. Cardoso, Microchem. J. 66 (1999) 273.

[15] A. Preto, M.R. Milani, A.A. Cardoso, J. Environ. Monit. 2 (2000) 566.

[16] I.M. Kolthoff, E.B. Sandell, Textbook of Quantitative Inorganic Analysis, 3rd ed., The Macmillan Company, New York, 1959, p. 651.

[17] H. Liu, P.K. Dasgupta, Anal. Chim. Acta 326 (1996) 13.

[18] K. Sonne, P.K. Dasgupta, Anal. Chem. 63 (1991) 427.
[19] P.K. Dasgupta, S. Dong, Atmos. Environ. 20 (1986) 565.

[20] K.T. Knapp, W.R. Pierson, P.K. Dasgupta, et al., in: J.P. Lodge (Ed.), Methods of Air Sampling and Analysis, 3rd ed., Lewis, Chelsea, MI, 1989, p. 523.

[21] H. Liu, P.K. Dasgupta, H.J. Zheng, Talanta 40 (1986) 1331.

[22] J.W. Strutt, Philos. Mag. 41 (1871) 107.

[23] F.P. Hochgesang, in: I.M. Kolthoff, P.J. Elving (Eds.), Treatise on Analytical Chemistry, Part I, vol. 5, Interscience Publishers, 1964, p. 3289.

[24] S. Liu, P.K. Dasgupta, Anal. Chem. 67 (1995) 2042.

[25] F.W Billmeyer Jr, in: I.M. Kolthoff, P.J Elving (Eds.), Treatise on Analytical Chemistry, Part I, vol. 5, Interscience Publishers, 1964, p. 2839.

[26] H.J. Backström, Z. Phys. Chem. 25B (1934) 122. 